\title{
Severity of Liver Injury and Atherogenic Lipid Profile in Children With Nonalcoholic Fatty Liver Disease
}

\author{
VALERIO NOBILI, NAIM ALKHOURI, ANDREA BARTULI, MELANIA MANCO, ROCIO LOPEZ, ANNA ALISI,
} AND ARIEL E. FELDSTEIN

\begin{abstract}
Liver Unit [V.N., M.M., A.A.], and Rare Diseases Unit [A.B.], “Bambino Gesù” Children's Hospital and Research Institute, Rome 00165, Italy; Departments of Pediatric Gastroenterology [N.A., A.E.F.], Quantitative Health Sciences [R.L.], and Cell Biology [A.E.F.], Cleveland Clinic, Cleveland, Ohio 44195
\end{abstract}

\begin{abstract}
Nonalcoholic fatty liver disease (NAFLD) is the hepatic manifestation of the metabolic syndrome. The aim of this study was to assess the relationship between severity of liver injury and atherogenic lipid profile in a large group of children with NAFLD. A total of 118 consecutive children with biopsy-proven NAFLD were included. Patients underwent extensive metabolic profiling. The NAFLD activity and fibrosis scores showed a significant positive correlation with triglyceride/HDL, total cholesterol/HDL, and LDL/HDL ratios $(p<0.05)$ but not with apolipoprotein B/apolipoprotein A-1 ratio $(p=0.58)$. After adjusting for BMI, homeostatic model assessment, impaired glucose tolerance, and presence of metabolic syndrome, both the NAFLD activity score and stage of fibrosis remained independent predictors of proatherogenic lipid profile. All lipid ratios, except for apolipoprotein B/apolipoprotein A-1, were found to be markedly higher in children with nonalcoholic steatohepatitis compared with those with simple steatosis or borderline disease ( $p$ $<0.05$ ). This study shows for the first time that in children with NAFLD, the severity of liver injury is strongly associated with the presence of a more atherogenic lipid profile, having potential significant diagnostic and therapeutic implications. (Pediatr Res 67: 665-670, 2010)
\end{abstract}

$\mathrm{N}$ onalcoholic fatty liver disease (NAFLD) is currently the most common form of chronic liver disease in both children and adults and threatens to become a serious public health problem $(1,2)$. It is estimated that one in three adults and one in 10 children or adolescents in the United States have NAFLD $(3,4)$, which is tightly associated with obesity and insulin resistance (IR). NAFLD encompasses a spectrum of disease ranging from simple steatosis in which triglycerides (TG) accumulate in hepatocytes in the absence of evidence for liver damage or inflammation to nonalcoholic steatohepatitis (NASH), defined by the association of lipid accumulation with evidence of cellular damage, inflammation, and different degrees of fibrosis (5).

At present, the most commonly used noninvasive tests to diagnose NAFLD include measurement of serum aminotransferases and liver ultrasound, alone or in combination $(6,7)$. Although these tools are in general useful for the diagnostic of NAFLD, they lack specificity and sensitivity to distinguish NASH from simple steatosis and determine the presence and

Received September 24, 2009; accepted January 17, 2010.

Correspondence: Valerio Nobili, M.D., Liver Unit, Bambino Gesu' Children's Hospital and Research Institute, S. Onofrio 4 Square, 00165 Rome, Italy; e-mail: nobili66@yahoo.it

Supported by “Bambino Gesù” Children's Hospital and Research Institute, Rome, Italy. extent of liver damage and fibrosis $(6,7)$. Thus, currently, a liver biopsy remains the only reliable way to distinguish NASH from simple steatosis.

A growing body of evidence from epidemiologic studies in both adult and children using either liver enzymes or ultrasound as surrogate markers of NAFLD has established NAFLD as an independent predictor for development of metabolic syndrome (MS), diabetes, and cardiovascular (CV) disease (8-11). CV disease remains the leading cause of death in the adult population, and it has been shown that the atherosclerotic process begins in childhood (12). In the Bogalusa heart study in children, investigators found that the extent to which the intimal surface was covered with atherosclerotic lesions was significantly associated with elevation of concentrations of total cholesterol (TC), LDL, TG, and lower concentration of HDL. Ratios of cholesterol ester-rich lipoprotein levels (TC/HDL and LDL/HDL) are well-established predictors of CV disease (13). More recently, the TG/HDL ratio has been shown to be a strong predictor of MS and CV disease $(14,15)$. In addition, recent studies have suggested that apolipoprotein $\mathrm{B}$ (ApoB)/apolipoprotein A-1 (ApoA-1) ratio may be a good predictor of CV risk and the presence of MS and IR (16).

Recently, experimental studies have suggested that the presence and degree of liver damage and inflammation may play a crucial pathogenic role in the development of these conditions (17-19). However, there is currently a paucity of data regarding the possible association between liver histopathological changes and atherogenic risk in both adults and children. Thus, the aim of this study was to assess the relationship between severity of liver injury and atherogenic lipid profile in a large, well-characterized group of children with biopsy-proven NAFLD using different lipid ratios as predictors of $\mathrm{CV}$ risk.

\section{PATIENTS AND METHODS}

Patients. A total of 118 consecutive patients diagnosed with NAFLD (60 male and 58 female) seen at Bambino Gesù Children's Hospital from January 2005 to April 2008 were included in the study. The study was approved by the Ethics Committee of the Bambino Gesù Children's Hospital and Research

Abbreviations: ApoB, apolipoprotein B; ApoA-1, apolipoprotein A-1; MS, metabolic syndrome; NAFLD, nonalcoholic fatty liver disease; NASH, nonalcoholic steatohepatitis; NAS, NAFLD activity score; OGTT, oral glucose tolerance test; TG, triglycerides; TC, total cholesterol 
Institute, Rome, Italy. Informed consent was obtained from each patient or responsible guardian.

Inclusion criteria were persistently elevated serum aminotransferase levels, diffusely echogenic liver on imaging studies suggestive of fatty liver, and biopsy consistent with the diagnosis of NAFLD (20-22). Exclusion criteria were hepatic virus infections (Hepatitis A-E and G, cytomegalovirus, and Epstein-Barr virus), alcohol consumption, history of parenteral nutrition, and use of drugs that are known to induce steatosis (e.g. valproate, amiodarone, or prednisone) or to affect body weight and carbohydrate metabolism. Autoimmune liver disease, metabolic liver disease, Wilson's disease, and alpha-1antitrypsin-associated liver disease were ruled out using standard clinical, laboratory, and histologic criteria.

The BMI and BMI $Z$ score were calculated $(23,24)$. MS was defined as the presence of $\geq 3$ of the following five criteria (25): abdominal obesity (defined by waist circumference $\geq 90$ th percentile for age) (26); hypertriglyceridemia as TG $>95$ th percentile for age, sex, and race (27); low-HDL cholesterol as concentrations $<5$ th percentile for age and sex (27); elevated blood pressure as systolic or diastolic blood pressure $>95$ th percentile for age and sex (28); and impaired fasting glucose or known type 2 diabetes mellitus (as defined in the next section). Physical maturation was assessed by two expert physicians (an endocrinologist M.M. and a pediatrician V.N.) according to Tanner (29). Subjects were divided into Tanner stage (T) as follows: prepubertal (T1), early pubertal (T2), midpubertal (T3-4), and late pubertal (T5).

Evaluation of lipid profiles. Serum TG (intra-assay coefficient of variation $1.5 \%$ and interassay coefficient of variation $1.8 \%$ ), TC (intra-assay coefficient of variation $0.8 \%$ and interassay coefficient of variation $1.7 \%$ ), and HDL cholesterol (intra-assay coefficient of variation $0.95 \%$ and interassay coefficient of variation $1.3 \%$ ) were measured by routine colorimetric assays (Roche/Hitachi Modular P/D, Can 433, Milan, Italy). LDL cholesterol was calculated using the Friedewald formula (LDL $=\mathrm{TC}-\mathrm{TG} / 5-\mathrm{HDL}$ ). Serum ApoA-1 and ApoB levels were measured using ADVIA-1650 Chemistry System (Bayer Corporation, Tarrytown, NY). None of our patients was on lipid-lowering medications at the time of lipid profiles measurements.

Glucose metabolism and insulin sensitivity. A 2-h oral glucose tolerance test (OGTT) was performed with the standard $1.75 \mathrm{~g}$ of glucose per $\mathrm{kg}$ of body weight or a maximum of $75 \mathrm{~g}$. Glucose tolerance status was determined according to the classification of the American Diabetes Association in which fasting plasma glucose levels up to $99 \mathrm{mg} / \mathrm{dL}$ are considered normal; impaired fasting glucose is defined by a fasting plasma glucose of $100-125 \mathrm{mg} / \mathrm{dL}$; impaired glucose tolerance is defined by a 2-h plasma glucose of 140-199 $\mathrm{mg} / \mathrm{dL}$; and diabetes mellitus is defined by a fasting plasma glucose $\geq 126$ $\mathrm{mg} / \mathrm{dL}$ or a 2 -h plasma glucose $\geq 200 \mathrm{mg} / \mathrm{dL}$ (30,31). Plasma glucose was measured in triplicate by the glucose oxidase technique on a Beckman glucose analyzer (Beckman, Fullerton, CA); and plasma insulin was measured by a specific radioimmunoassay (MYRIA Technogenetics, Milan, Italy).

The degrees of IR and sensitivity were determined, respectively, by the homeostatic model assessment insulin resistance using the formula: insulin resistance $=($ insulin $\times$ glucose $) / 22.5$ and by the insulin sensitivity index derived from OGTT using the formula: insulin sensitivity index $=[10,000 /$ square root of (fasting glucose $\times$ fasting insulin $) \times$ (mean glucose $\times$ mean insulin during OGTT)] $(32,33)$.

Liver histology. The clinical indication for biopsy was either to assess the presence of NASH and degree of fibrosis or other likely independent or competing liver diseases. Liver biopsy was performed in all children, after an overnight fast, using an automatic core biopsy 18-gauge needle (Biopince, Amedic, Sweden) under general anesthesia and ultrasound guidance. A Sonoline Omnia Ultrasound machine (Siemens, Germany) with a $5-\mathrm{MHz}$ probe (5.0 C 50, Siemens) was used. Two biopsy passes within different liver segments were performed for each subject. The length of liver specimen (in millimetres) was recorded. Only samples with a length $\geq 15 \mathrm{~mm}$ and including at least 10-11 complete portal tracts were considered adequate for the purpose of the study (34). Biopsies were evaluated by a single liver pathologist. Biopsies were routinely processed (i.e. formalin fixed and paraffin embedded). Sections of liver tissue, $5-\mu \mathrm{m}$ thick, were stained with hematoxylin and eosin, Van Gieson, Periodic acid-Schiff, digested Stain, and Prussian blue stain. Immunohistochemical staining with antibodies to alpha-1antitrypsin was used to exclude alpha-1-antitrypsin deficiency-associated liver disease. Liver biopsy features were graded according to the NAFLD activity scoring (NAS) system proposed by Kleiner et al. (35). Briefly, grade of steatosis was scored as $0=<5 \%, 1=5-33 \%, 2=>33-66 \%$, and $3=$ $>66 \%$; grade of lobular inflammation was scored as $0=$ no foci, $1=<2$ foci $/ 200 \times$ field, $2=2-4$ foci $/ 200 \times$ field, and $3=>4$ foci $/ 200 \times$ field; and grade of ballooning was scored as $0=$ none, $1=$ few ballooning cells, and $2=$ many cells/prominent ballooning. The grade of steatosis $(0-3)$, lobular inflammation $(0-3)$, and ballooning $(0-2)$ were then combined to determine the NAFLD activity score $(0-8)$ as proposed. Fibrosis was scored as $0=$ none; 1 = periportal or perisinusoidal fibrosis; $2=$ perisinusoidal and portal/periportal fibrosis; $3=$ bridging fibrosis; and $4=$ cirrhosis $(35)$. The liver biopsy samples were then classified as definitive NASH (unequivocally fulfils previously described criteria for steatohepatitis), borderline diagnosis (some but not all histologic features of steatohepatitis), and simple steatosis (isolated fat deposition in hepatocytes).

Statistical analysis. Descriptive statistics were computed for all factors. These included medians and percentiles for continuous factors and frequencies for categorical variables. Spearman correlation coefficients were estimated to evaluate correlations between lipid ratios (including TG/HDL, cholesterol/HDL, LDL/HDL, non-HDL cholesterol/HDL, and ApoB/A ratios) and several continuous and ordinal factors. In addition, Wilcoxon rank-sum tests were used to assess associations between lipid ratios and several categorical variables such as MS. ANOVA was performed to study the association between NAFLD and lipid ratios; the natural logarithm transformation of each lipid ratio was modeled as the outcome, and post hoc analyses were performed using Bonferroni's adjustment for multiple comparisons (36). In addition, analysis of covariance was performed to adjust for potential confounders such as age, sex, puberty development, and MS components. A $p<0.05$ was considered statistically significant. SAS version 9.2 software (The SAS Institute, Cary, NC) and R version 2.4.1 software (The R Foundation for Statistical Computing, Vienna, Austria) was used for all analyses.

\section{RESULTS}

Patient characteristics. The main anthropometric, clinical, and laboratory data of our patient population are summarized in Table 1 . The median age was 11 years (interquartile range, $9-13$ years), there was an equal proportion of boys and girls, and two-thirds were obese. The features of the MS were commonly present with more than two-thirds having a waist circumference $\geq 90$ th percentile, 29 patients (24.6\%) presented with hypertriglyceridemia, 28 (23.7\%) with low-HDL cholesterol, 30 (25.4\%) had glucose intolerance, and 27 patients (22.9\%) suffered from hypertension. One-hundred twelve children (95\%) presented with at least one feature of the MS, whereas overt MS (i.e. $\geq 3$ features) was present in 26 children (22\%) (Table 2).

Liver histology. The main liver biopsy features are summarized in Table 2. Steatosis was present in all cases, and it was of moderate-to-severe grade in most patients. Lobular inflammation was of mild severity in most children. Fifty-five percent of cases revealed ballooning of the hepatocytes that was in most cases of grade 1 (few balloon cells). The median NAS was 4 (interquartile range, 3-5). Seventeen patients (14.4\%) were classified as simple steatosis, 58 patients $(49.1 \%)$ as borderline disease, and 43 patients $(36.4 \%)$ as definitive NASH. Some degree of fibrosis was present in $64 \%$ of cases including mild fibrosis (stage 1) in 58 patients (49\%) and moderate fibrosis (stage 2) in 11 patients (9\%), whereas 6 patients (5\%) showed advanced fibrosis (stage 3). No patient had established cirrhosis. Interface hepatitis or other features suggestive of autoimmune hepatitis were not present in any of the cases.

Association of severity of liver disease with atherogenic lipid profile in children with NAFLD. To study the potential association of severity of liver disease with atherogenic risk in children with NAFLD, we first assessed the correlation of the individual histologic features (steatosis, inflammation, ballooning of hepatocytes, and fibrosis) and the NAS with established predictors of atherogenic risk profile. The NAS and its individual components showed a significant positive correlation with TG/HDL, TC/HDL, and LDL/ HDL ratios $(p<0.05$ for all) but not with ApoB/ApoA-1 ratio ( $p=0.58$; Table 3 ). Similarly, a positive correlation was found between these scores and stage of fibrosis (Table 
Table 1. Demographic, clinical, and laboratory features in children with NAFLD

\begin{tabular}{|c|c|c|c|c|}
\hline Factor & $(N=118)$ & Male $(N=60)$ & Female $(N=58)$ & $p$ \\
\hline Male & $60(50.9)$ & $60(100)$ & $0(0)$ & - \\
\hline Age (yr) & $11(9.3,12.7)$ & $11.1(10.0,13.2)$ & $10.1(9.0,12.1)$ & 0.026 \\
\hline BMI $\left(\mathrm{kg} / \mathrm{m}^{2}\right)$ & $24.4(22.4,28.7)$ & $25.0(22.8,28.0)$ & $24.4(21.2,28.9)$ & 0.38 \\
\hline Waist circumference $(\mathrm{cm})$ & $87.5(80,95)$ & $89.0(81.0,96.0)$ & $85.0(79.0,94.0)$ & 0.16 \\
\hline Fasting blood glucose (mg/dL) & $78.5(74,84)$ & $80.0(76.0,85.5)$ & $78.0(73.0,83.0)$ & 0.16 \\
\hline Fasting insulin $(\mu \mathrm{U} / \mathrm{mL})$ & $10.6(6.9,16)$ & $9.1(6.8,13.4)$ & $11.3(7.8,17.1)$ & 0.092 \\
\hline HOMA-IR & $2(1.4,3)$ & $1.8(1.3,2.7)$ & $2.2(1.4,3.2)$ & 0.16 \\
\hline ISI & $3.9(3,5.5)$ & $4.0(3.1,6.2)$ & $3.8(2.6,5.0)$ & 0.14 \\
\hline Cholesterol (mg/dL) & $176(151,190)$ & $176.0(156.0,189.0)$ & $174.5(145.0,192.0)$ & 0.8 \\
\hline Triglycerides (mg/dL) & $88.5(71,116)$ & $88.5(68.5,112.5)$ & $88.5(74.0,131.0)$ & 0.29 \\
\hline $\operatorname{HDL}(\mathrm{mg} / \mathrm{dL})$ & $51(42,61)$ & $54.0(45.0,66.0)$ & $51.0(41.0,60.0)$ & 0.27 \\
\hline $\mathrm{LDL}(\mathrm{mg} / \mathrm{dL})$ & $99.4(77.0,116.8)$ & $99.4(81.6,115.4)$ & $99.4(76.0,123.8)$ & 0.75 \\
\hline Systolic blood pressure $(\mathrm{mm} \mathrm{Hg})$ & $110(101,120)$ & $110.0(101.5,120.5)$ & $106.5(100.0,120.0)$ & 0.36 \\
\hline Diastolic blood pressure $(\mathrm{mm} \mathrm{Hg})$ & $70(62,76)$ & $70.0(64.0,78.0)$ & $70.0(61.0,73.0)$ & 0.2 \\
\hline $\operatorname{ALT}(\mathrm{IU} / \mathrm{L})$ & $61(50,78)$ & $61.0(45.0,78.0)$ & $62.0(51.0,88.0)$ & 0.44 \\
\hline AST (IU/L) & $45(34,67)$ & $48.5(35.5,63.0)$ & $45.0(34.0,67.0)$ & 0.96 \\
\hline GGT (IU/L) & $22(18,27)$ & $22.0(18.0,29.5)$ & $22.0(18.0,24.0)$ & 0.8 \\
\hline Apolipoprotein A (mg/dL) & $109(85,124)$ & $110.0(95.0,130.0)$ & $100.5(80.0,121.0)$ & 0.059 \\
\hline Apolipoprotein B (mg/dL) & $77(59,87)$ & $80.0(55.5,87.5)$ & $72.0(59.0,85.0)$ & 0.86 \\
\hline
\end{tabular}

Values presented as $N(\%)$ or median (P25, P75).

HOMA-IR, homeostatic model assessment insulin resistance; ISI, insulin sensitivity index; ALT, alanine aminotransferase; AST, aspartate aminotransferase.

Table 2. Metabolic syndrome and histological characteristics in children with NAFLD

\begin{tabular}{|c|c|c|c|c|}
\hline Factor & $(N=118)$ & Male $(N=60)$ & Female $(N=58)$ & $p$ \\
\hline Waist circumference $\geq 90$ th percentile & $91(77.1)$ & $47(78.3)$ & $44(75.9)$ & 0.75 \\
\hline Hypertension & $27(22.9)$ & $11(18.3)$ & $16(27.6)$ & 0.23 \\
\hline Elevated triglycerides & $29(24.6)$ & $13(21.7)$ & $16(27.6)$ & 0.46 \\
\hline Low HDL & $28(23.7)$ & $14(23.3)$ & $14(24.1)$ & 0.92 \\
\hline Glucose intolerance & $30(25.4)$ & $14(23.3)$ & $16(27.6)$ & 0.6 \\
\hline \multicolumn{5}{|l|}{ Number of MS components } \\
\hline 0 & $6(5.1)$ & $3(5.0)$ & $3(5.2)$ & 0.23 \\
\hline 1 & $48(40.7)$ & $28(46.7)$ & $20(34.5)$ & \\
\hline 2 & $38(32.2)$ & $18(30.0)$ & $20(34.5)$ & \\
\hline 3 & $23(19.5)$ & $9(15.0)$ & $14(24.1)$ & \\
\hline 4 & $3(2.5)$ & $2(3.3)$ & $1(1.7)$ & \\
\hline \multicolumn{5}{|l|}{ Steatosis } \\
\hline$<5 \%$ & $1(0.9)$ & $0(0.0)$ & $1(1.7)$ & 0.59 \\
\hline $5-33 \%$ & $21(17.8)$ & $12(20.0)$ & $9(15.5)$ & \\
\hline $34-65 \%$ & $68(57.6)$ & $35(58.3)$ & $33(56.9)$ & \\
\hline$\geq 66 \%$ & $28(23.7)$ & $13(21.7)$ & $15(25.9)$ & \\
\hline \multicolumn{5}{|l|}{ Inflammation } \\
\hline None & $8(6.8)$ & $4(6.7)$ & $4(6.9)$ & 0.54 \\
\hline$<2$ under $20 \times$ & $77(65.3)$ & $41(68.3)$ & $36(62.1)$ & \\
\hline $2-4$ under 20 & $33(28.0)$ & $15(25.0)$ & $18(31.0)$ & \\
\hline \multicolumn{5}{|l|}{ Ballooning } \\
\hline None & $53(44.9)$ & $25(41.7)$ & $28(48.3)$ & 0.9 \\
\hline Few & $43(36.4)$ & $26(43.3)$ & $17(29.3)$ & \\
\hline Many & $22(18.6)$ & $9(15.0)$ & $13(22.4)$ & \\
\hline \multicolumn{5}{|l|}{ Fibrosis } \\
\hline 0 & $43(36.4)$ & $25(41.7)$ & $18(31.0)$ & 0.39 \\
\hline 1 & $58(49.2)$ & $26(43.3)$ & $32(55.2)$ & \\
\hline 2 & $11(9.3)$ & $6(10.0)$ & $5(8.6)$ & \\
\hline 3 & $6(5.1)$ & $3(5.0)$ & $3(5.2)$ & \\
\hline
\end{tabular}

Values presented as $N(\%)$.

3 and Fig. 1). After adjusting for potential confounders including BMI, homeostatic model assessment index, impaired glucose tolerance, and presence of MS, both NAS and stage of fibrosis remained independent predictors of an atherogenic lipid profile (Table 4).

All lipid ratios, except for ApoB/ApoA-1, were found to be markedly higher in children with established NASH compared with those patients with simple steatosis or borderline disease $(p<0.05$ for all; Fig. 2). There was no evidence to suggest a significant difference in lipid ratios between patients with simple steatosis and those with borderline disease. Thus, these data strongly suggest that in children with NAFLD, NASH, and fibrosis are at particular risk for $\mathrm{CV}$ disease associated with an atherogenic lipid profile. 
Table 3. Correlations between clinical characteristics, liver histological features, MS, and lipid markers of CV risk

\begin{tabular}{|c|c|c|c|c|c|c|c|c|c|}
\hline \multirow[b]{2}{*}{ Factor } & \multicolumn{3}{|c|}{ Triglycerides/HDL ratio } & \multicolumn{3}{|c|}{ Cholesterol/HDL ratio } & \multicolumn{3}{|c|}{ LDL/HDL ratio } \\
\hline & $\rho$ & $95 \% \mathrm{CI}$ & $P$ & $\rho$ & $95 \% \mathrm{CI}$ & $P$ & $\rho$ & $95 \% \mathrm{CI}$ & $P$ \\
\hline BMI & 0.09 & $(-0.10,0.27)$ & 0.35 & 0.04 & $(-0.14,0.23)$ & 0.66 & 0.03 & $(-0.16,0.21)$ & 0.78 \\
\hline WC & 0.01 & $(-0.18,0.19)$ & 0.95 & -0.11 & $(-0.30,0.07)$ & 0.23 & -0.11 & $(-0.30,0.07)$ & 0.23 \\
\hline Fasting glucose & -0.03 & $(-0.21,0.16)$ & 0.78 & 0.11 & $(-0.07,0.29)$ & 0.24 & 0.13 & $(-0.06,0.31)$ & 0.18 \\
\hline HOMA-IR & 0.02 & $(-0.17,0.20)$ & 0.85 & 0.06 & $(-0.13,0.24)$ & 0.54 & 0.07 & $(-0.11,0.25)$ & 0.45 \\
\hline ISI & -0.13 & $(-0.31,0.05)$ & 0.16 & -0.17 & $(-0.36,0.01)$ & 0.06 & -0.18 & $(-0.37,-0.00)$ & 0.047 \\
\hline Systolic BP & -0.02 & $(-0.20,0.17)$ & 0.86 & -0.12 & $(-0.30,0.07)$ & 0.21 & -0.12 & $(-0.30,0.07)$ & 0.21 \\
\hline Diastolic BP & 0.12 & $(-0.06,0.31)$ & 0.2 & -0.03 & $(-0.21,0.16)$ & 0.75 & -0.06 & $(-0.24,0.13)$ & 0.53 \\
\hline ALT & 0.29 & $(0.11,0.46)$ & 0.002 & 0.14 & $(-0.04,0.32)$ & 0.13 & 0.09 & $(-0.10,0.27)$ & 0.36 \\
\hline AST & 0.25 & $(0.07,0.42)$ & 0.008 & 0.15 & $(-0.03,0.33)$ & 0.11 & 0.11 & $(-0.08,0.29)$ & 0.26 \\
\hline GGT & 0.25 & $(0.07,0.43)$ & 0.007 & 0.14 & $(-0.05,0.32)$ & 0.14 & 0.09 & $(-0.09,0.28)$ & 0.31 \\
\hline NAS & 0.42 & $(0.25,0.59)$ & $<0.001$ & 0.33 & $(0.15,0.50)$ & $<0.001$ & 0.28 & $(0.10,0.45)$ & 0.003 \\
\hline Number of MS components & 0.49 & $(0.33,0.65)$ & $<0.001$ & 0.48 & $(0.32,0.64)$ & $<0.001$ & 0.36 & $(0.19,0.53)$ & $<0.001$ \\
\hline Steatosis & 0.31 & $(0.13,0.48)$ & $<0.001$ & 0.27 & $(0.09,0.45)$ & 0.003 & 0.23 & $(0.06,0.41)$ & 0.011 \\
\hline Inflammation & 0.32 & $(0.15,0.50)$ & $<0.001$ & 0.31 & $(0.13,0.49)$ & $<0.001$ & 0.28 & $(0.10,0.46)$ & 0.002 \\
\hline Ballooning & 0.34 & $(0.17,0.52)$ & $<0.001$ & 0.18 & $(0.00,0.37)$ & 0.046 & 0.14 & $(-0.04,0.32)$ & 0.013 \\
\hline Fibrosis & 0.35 & $(0.17,0.52)$ & $<0.001$ & 0.27 & $(0.09,0.45)$ & 0.003 & 0.24 & $(0.06,0.42)$ & 0.01 \\
\hline
\end{tabular}

H0: $\rho=0$.

WC, waist circumference; HOMA-IR, homeostatic model assessment insulin resistance; BP, blood pressure; ALT, alanine aminotransferase; AST, aspartate aminotransferase; GGT, gamma glutamyl transpeptidase.
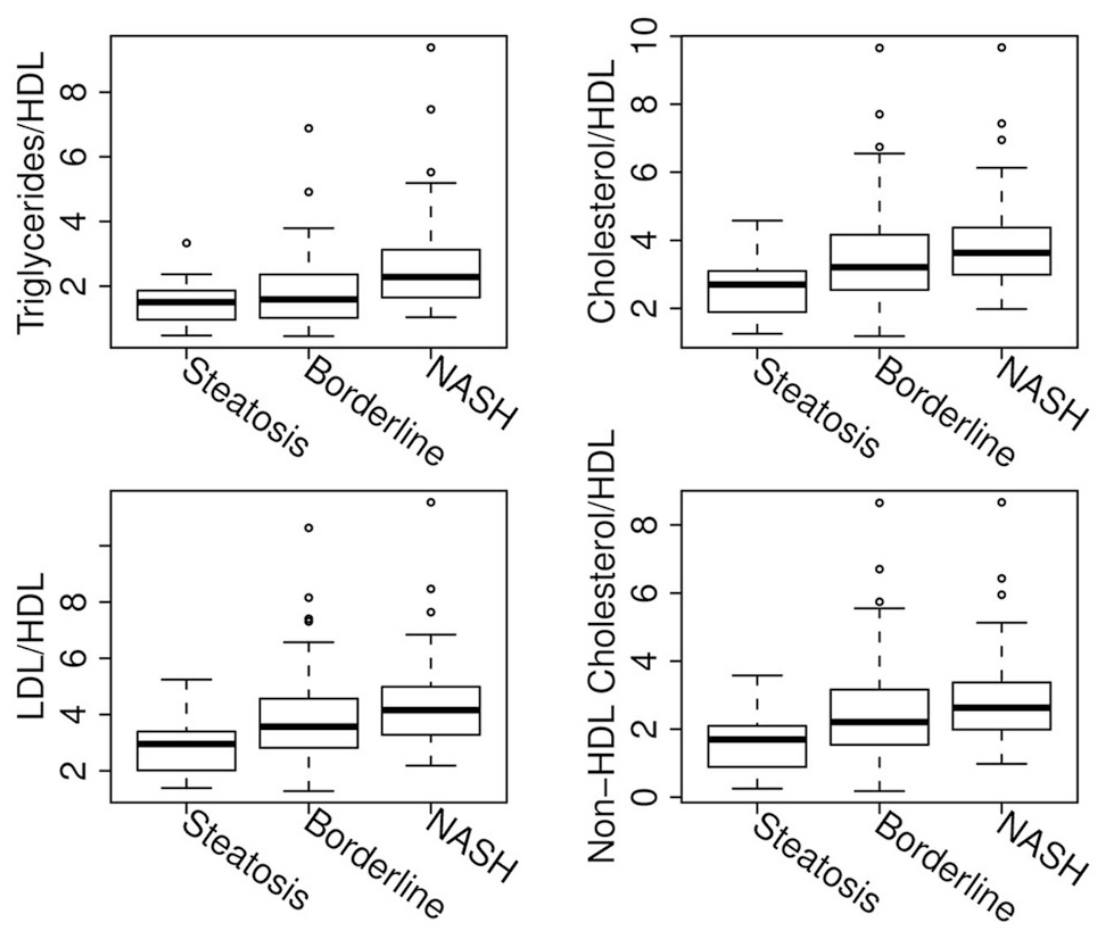

Figure 1. Association between lipid markers of CV risk and liver histology. Vertical axis represents the lipid ratio, and horizontal axis represents patient groups according the NAS. The box represents the interquartile range (the 25 th and 75th percentiles) from the median (horizontal line) and the bars represent the $95 \%$ confidence interval. All lipid ratios were significantly increased in patients with definitive NASH compared with patients with simple steatosis or borderline diagnosis.

\section{DISCUSSION}

The principal findings of this study relate to the link between severity of liver disease and atherogenic risk in children with NAFLD. The results show that the degree of liver injury and stage of fibrosis positively correlates with established markers of atherogenic risk. The association between liver damage and an atherogenic profile is independent of obesity, IR, and the presence of MS in children with NAFLD. The atherogenic profile is particularly prominent in patients with established NASH diagnosis as opposed to those with simple steatosis. Taken together, these results suggest a significant effect of the severity of NAFLD on $\mathrm{CV}$ risk in children.
With the growing prevalence of childhood obesity and overweight, pediatric NAFLD has become the most frequent chronic liver disease in children and adolescents in industrialized countries. A recent large autopsy study found that 9.6\% of Americans aged 2 to $19 \mathrm{y}$ have NAFLD, and this figure increased to $38 \%$ among those who were obese (4). Similar high figures have been reported among children from other countries in Europe and Asia $(37,38)$. Currently, a liver biopsy remains the gold standard for NAFLD diagnosis (39). A liver biopsy provides key information regarding the degree of liver damage and remains the only reliable way of distinguishing NASH from simple steatosis. 
Table 4. NAFLD, fibrosis, and lipid ratios: ANCOVA

\begin{tabular}{lccc}
\hline \multicolumn{1}{c}{ Factor } & Triglyceride/HDL & Cholesterol/HDL & LDL/HDL \\
\hline NAFLD & & & \\
$\quad$ Steatosis & $1.35(1.00-1.83)$ & $2.62(2.13-3.22)$ & $1.24(0.85-1.81)$ \\
Borderline & $1.54(1.28-1.85)$ & $3.36(2.96-3.82)$ & $2.05(1.60-2.64)$ \\
NASH & $2.38(1.96-2.89)$ & $3.76(3.29-4.30)$ & $2.29(1.80-2.91)$ \\
$p$ & 0.0001 & 0.007 & 0.01 \\
Fibrosis & & & \\
0 & $1.33(1.06-1.68)$ & $2.87(2.46-3.35)$ & $1.50(1.12-2.03)$ \\
1 & $1.90(1.58-2.28)$ & $3.52(3.11-3.99)$ & $2.14(1.69-2.71)$ \\
2 & $2.06(1.44-2.95)$ & $3.71(2.91-4.73)$ & $2.19(1.43-3.35)$ \\
3 & $2.78(1.73-4.49)$ & $4.23(3.06-5.84)$ & $2.72(1.54-4.80)$ \\
$p$ & 0.004 & 0.026 & 0.05 \\
\hline
\end{tabular}

Values presented as mean (95\% confidence limits). $p$ values are presented correspond to ANCOVA adjusting for age, sex, pubertal development, WC, HTN, and IGT.

ANCOVA, analysis of covariance; IGT, impaired glucose tolerance.

There is a growing body of evidence suggesting an association between NAFLD and CV disease (8-11,40,41). Recent longitudinal long-term studies from both the United States and Europe have demonstrated that $\mathrm{CV}$ mortality is increased in adult patients with NAFLD (42-44). Moreover, these studies have suggested that the increased in CV mortality occurs in patients with NASH and fibrosis but not in those with simple steatosis.

Several cross-sectional studies have shown that patients with NAFLD have increased carotid artery atherosclerosis with enlarged intima-media thickness and increased prevalence of carotid plaques (45-47). Although others have demonstrated a link between NAFLD and the presence of endothelial dysfunction as assessed by brachial artery flow-mediated vasodilation (48), two recent studies in children attempted to evaluate the relationship between early atherosclerosis and NAFLD. Similar to adults, children with NAFLD were found to have increased carotid intima-media thickness compared with matched controls $(49,50)$. A central limitation of these studies was the fact that the diagnosis of NAFLD was based on evaluation of liver enzymes or liver ultrasound; thus, the potential impact of the spectrum of NAFLD such as the presence of NASH versus simple steatosis and the relationship between the extend of liver damage and CV risk could not be assessed.

Recent experimental studies in mice have suggested that hepatic inflammation and hepatic IR may contribute to the devel- opment of dyslipidemia and increased susceptibility to atherosclerosis (17-19). We were able to show that this is also true in children with NAFLD. Our results identified a potential link between severity of liver damage and atherogenic lipid profile in children with biopsy-proven NAFLD. Indeed, we showed that the presence of NASH and fibrosis on liver biopsy in these patients correlates with lipid markers of atherogenic and $\mathrm{CV}$ risk. This association was independent of obesity, IR, and the presence of MS. This result is not surprising, because not all our patients are obese, suggesting the possible involvement of other risk factors associated with NAFLD, including genetic predisposition and dietary habits. Moreover, the TG/HDL ratio was the best lipid ratio to predict the presence of advanced liver disease (higher NAS and fibrosis stage). This is a novel finding and if validated in other studies, the TG/HDL ratio could help in selecting NAFLD patients with higher risk for disease progression who might benefit from liver biopsy and more aggressive follow-up. The ApoB/ApoA-1 ratio did not show the same correlation with liver histology as the other lipid ratios that may be explained by the fact that the liver is the major site for producing these lipoproteins.

The main strengths of our study are the inclusion of a large group of consecutively recruited children with liver biopsyproven NAFLD with the full spectrum of disease along with an extensive characterization of their metabolic profile. However, our study has some limitations including the fact that patients were seen at a large referral tertiary care medical center. Although the results may be extrapolated to other similar medical centers, the results may not apply to children with NAFLD from the community. Second, most of our children were white, making it difficult to determine whether the association between NAFLD severity and atherogenic risk is different among other ethnic groups. Finally, whether chronic liver inflammation and injury is a primary mechanism promoting a proatherogenic dyslipidemia cannot be elucidated by this study and warrants further investigations in animal models. An increase in TG secretion form the liver in the form of LDL is likely responsible for the increase in serum TG concentrations commonly noted in patients with NAFLD (51).

In summary, our results reveal for the first time that the severity of liver injury in children with NAFLD is strongly
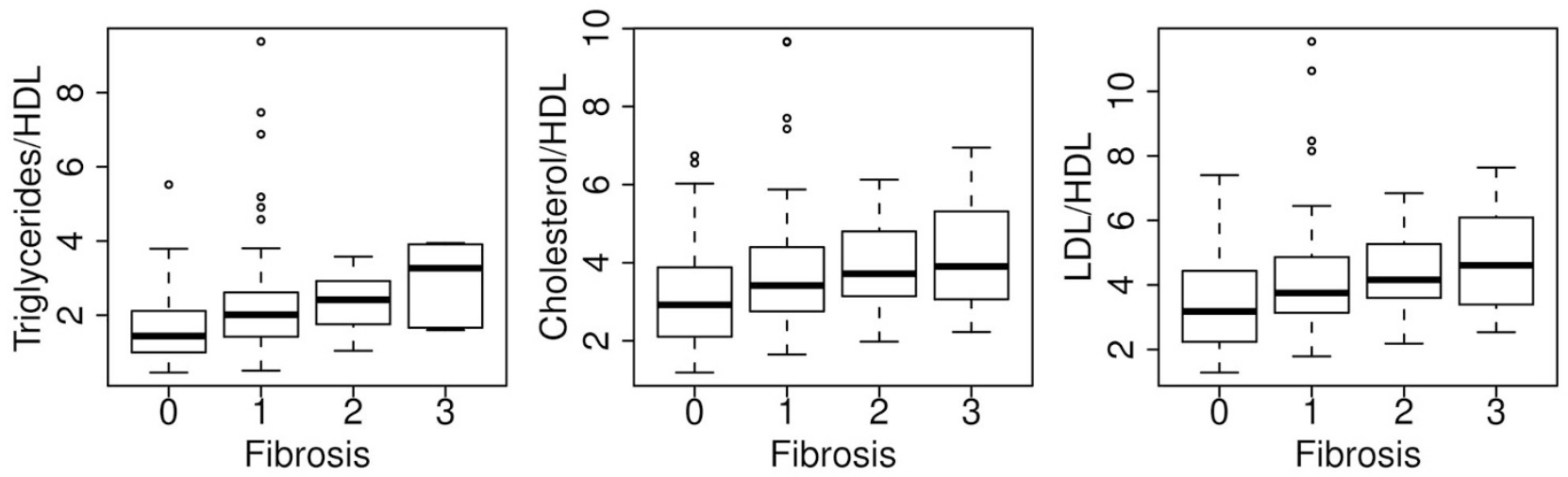

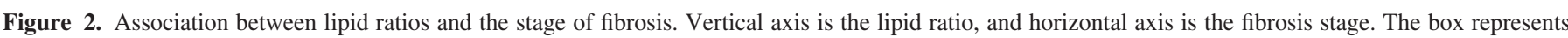

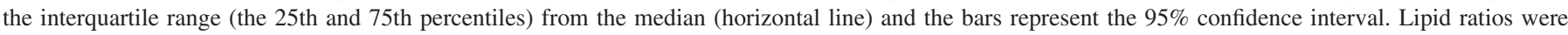
significantly higher in patients with moderate-to-severe fibrosis (stage 2-3) compared with those patients with no or mild fibrosis (stage 0-1). 
associated with increased atherogenic risk. Future long-term longitudinal studies are needed to evaluate development of CV disease in children with NAFLD and to determine the most appropriate therapeutic strategies for these patients. At this point, we recommend that atherogenic risk factors should be carefully evaluated and aggressively treated in patients with NAFLD, especially when the disease progresses to its more severe forms (NASH and fibrosis) as they may be associated with higher risk for early atherosclerosis and consequent $\mathrm{CV}$ disease.

\section{REFERENCES}

1. Wieckowska A, Feldstein AE 2005 Nonalcoholic fatty liver disease in the pediatric population: a review. Curr Opin Pediatr 17:636-641

2. Angulo P 2002 Nonalcoholic fatty liver disease. N Engl J Med 346:1221-1231

3. Browning JD, Szczepaniak LS, Dobbins R, Nuremberg P, Horton JD, Cohen JC, Grundy SM, Hobbs HH 2004 Prevalence of hepatic steatosis in an urban population in the United States: impact of ethnicity. Hepatology 40:1387-1395

4. Schwimmer JB, Deutsch R, Kahen T, Lavine JE, Stanley C, Behling C 2006 Prevalence of fatty liver in children and adolescents. Pediatrics 118:1388-1393

5. Brunt EM 2007 Pathology of fatty liver disease. Mod Pathol 20:S40-S48

6. Wieckowska A, Zein NN, Yerian LM, Lopez AR, McCullough AJ, Feldstein AE 2006 In vivo assessment of liver cell apoptosis as a novel biomarker of disease severity in nonalcoholic fatty liver disease. Hepatology 44:27-33

7. Wieckowska A, McCullough AJ, Feldstein AE 2007 Noninvasive diagnosis and monitoring of nonalcoholic steatohepatitis: present and future. Hepatology 46:582-589

8. Targher G, Franchini M, Guidi GC, Muggeo M, Lippi G 2007 Alanine aminotransferase as an independent predictor of incident nonalcoholic fatty liver disease. Clin Chem 53:1159; author reply 1159-1161

9. Schindhelm RK, Dekker JM, Nijpels G, Bouter LM, Stehouwer CD, Heine RJ, Diamant M 2007 Alanine aminotransferase predicts coronary heart disease events: a 10-year follow-up of the Hoorn Study. Atherosclerosis 191:391-396

10. Vento S, Nobili V 2008 Aminotransferases as predictors of mortality. Lancet 371:1822-1823

11. Goessling W, Massaro JM, Vasan RS, D'Agostino RB Sr, Ellison RC, Fox CS 2008 Aminotransferase levels and 20-year risk of metabolic syndrome, diabetes, and cardiovascular disease. Gastroenterology 135:1935-1944

12. Berenson GS, Srinivasan SR, Bao W, Newman WP III, Tracy RE, Wattigney WA 1998 Association between multiple cardiovascular risk factors and atherosclerosis in children and young adults. The Bogalusa Heart Study. N Engl J Med 338:1650-1656

13. Stampfer MJ, Sacks FM, Salvini S, Willett WC, Hennekens CH 1991 A prospective study of cholesterol, apolipoproteins, and the risk of myocardial infarction. N Engl J Med 325:373-381

14. McLaughlin T, Reaven G, Abbasi F, Lamendola C, Saad M, Waters D, Simon J, Krauss RM 2005 Is there a simple way to identify insulin-resistant individuals at increased risk of cardiovascular disease? Am J Cardiol 96:399-404

15. Quijada Z, Paoli M, Zerpa Y, Camacho N, Cichetti R, Villarroel V, Arata-Bellabarba G, Lanes R 2008 The triglyceride/HDL-cholesterol ratio as a marker of cardiovascular risk in obese children; association with traditional and emergent risk factors. Pediatr Diabetes 9:464-471

16. Sierra-Johnson J, Somers VK, Kuniyoshi FH, Garza CA, Isley WL, Gami AS, Lopez-Jimenez F 2006 Comparison of apolipoprotein-B/apolipoprotein-AI in subjects with versus without the metabolic syndrome. Am J Cardiol 98:1369-1373

17. Arbones-Mainar JM, Navarro MA, Carnicer R, Guillén N, Surra JC, Acín S, Guzmán MA, Sarría AJ, Arnal C, Aguilera MP, Jiménez A, Beltrán G, Uceda M, Osada J 2007 Accelerated atherosclerosis in apolipoprotein E-deficient mice fed Western diets containing palm oil compared with extra virgin olive oils: a role for small, dense high-density lipoproteins. Atherosclerosis 194:372-382

18. Biddinger SB, Hernandez-Ono A, Rask-Madsen C, Haas JT, Alemán JO, Suzuki R, Scapa EF, Agarwal C, Carey MC, Stephanopoulos G, Cohen DE, King GL, Ginsberg HN, Kahn CR 2008 Hepatic insulin resistance is sufficient to produce dyslipidemia and susceptibility to atherosclerosis. Cell Metab 7:125-134

19. Guillen N, Acin S, Navarro MA, Perona JS, Arbonés-Mainar JM, Arnal C, Sarría AJ, Surra JC, Carnicer R, Orman I, Segovia JC, Ruiz-Gutiérrez V, Osada J 2008 Squalene in a sex-dependent manner modulates atherosclerotic lesion which correlates with hepatic fat content in apoE-knockout male mice. Atherosclerosis 197:72-83

20. Nobili V, Manco M, Devito R, Ciampalini P, Piemonte F, Marcellini M 2006 Effect of vitamin $\mathrm{E}$ on aminotransferase levels and insulin resistance in children with non-alcoholic fatty liver disease. Aliment Pharmacol Ther 24:1553-1561

21. Nobili V, Manco M, Devito R, Di Ciommo V, Comparcola D, Sartorelli MR, Piemonte F, Marcellini M, Angulo P 2008 Lifestyle intervention and antioxidant therapy in children with nonalcoholic fatty liver disease: a randomized, controlled trial. Hepatology 48:119-128

22. Manco M, Bedogni G, Marcellini M, Devito R, Ciampalini P, Sartorelli MR, Comparcola D, Piemonte F, Nobili V 2008 Waist circumference correlates with liver fibrosis in children with non-alcoholic steatohepatitis. Gut 57:1283-1287

23. Kuczmarski RJ, Ogden CL, Grummer-Strawn LM, Flegal KM, Guo SS, Wei R, Mei Z, Curtin LR, Roche AF, Johnson CL 2000 CDC growth charts: United States. Adv Data 314:1-27
24. Cole TJ, Bellizzi MC, Flegal KM, Dietz WH 2000 Establishing a standard definition for child overweight and obesity worldwide: international survey. BMJ 320:1240-1243

25. Boney CM, Verma A, Tucker R, Vohr BR 2005 Metabolic syndrome in childhood: association with birth weight, maternal obesity, and gestational diabetes mellitus. Pediatrics 115:e290-e296

26. Fernandez JR, Redden DT, Pietrobelli A, Allison DB 2004 Waist circumference percentiles in nationally representative samples of African-American, EuropeanAmerican, and Mexican-American children and adolescents. J Pediatr 145:439-444

27. 1992 American Academy of Pediatrics. National cholesterol education program: report of the expert panel on blood cholesterol levels in children and adolescents. Pediatrics 89:525-584

28. 1987 Report of the Second Task Force on Blood Pressure Control in Children - 1987 Task Force on Blood Pressure Control in Children. National Heart, Lung, and Blood Institute, Bethesda, Maryland. Pediatrics 79:1-25

29. Tanner JM 1981 Growth and maturation during adolescence. Nutr Rev 39:43-55

30. Genuth S, Alberti KG, Bennett P, Buse J, Defronzo R, Kahn R, Kitzmiller J, Knowler WC, Lebovitz H, Lernmark A, Nathan D, Palmer J, Rizza R, Saudek C, Shaw J, Steffes M, Stern M, Tuomilehto J, Zimmet P; Expert Committee on the Diagnosis and Classification of Diabetes Mellitus 2003 Follow-up report on the diagnosis of diabetes mellitus. Diabetes Care 26:3160-3167

31. Genuth S 2003 Lowering the criterion for impaired fasting glucose is in order. Diabetes Care 26:3331-3332

32. Matsuda M, DeFronzo RA 1999 Insulin sensitivity indices obtained from oral glucose tolerance testing: comparison with the euglycemic insulin clamp. Diabetes Care 22:1462-1470

33. Abdul-Ghani MA, Matsuda M, Balas B, DeFronzo RA 2007 Muscle and liver insulin resistance indexes derived from the oral glucose tolerance test. Diabetes Care 30:89-94

34. Poynard T, Halfon P, Castera L, Charlotte F, Le Bail B, Munteanu M, Messous D, Ratziu V, Benhamou Y, Bourlière M, De Ledinghen V; FibroPaca Group 2007 Variability of the area under the receiver operating characteristic curves in the diagnostic evaluation of liver fibrosis markers: impact of biopsy length and fragmentation. Aliment Pharmacol Ther 25:733-739

35. Kleiner DE, Brunt EM, Van Natta M, Behling C, Contos MJ, Cummings OW, Ferrell LD, Liu YC, Torbenson MS, Unalp-Arida A, Yeh M, McCullough AJ, Sanyal AJ; Nonalcoholic Steatohepatitis Clinical Research Network 2005 Design and validation of a histological scoring system for nonalcoholic fatty liver disease. Hepatology 41:1313-1321

36. Bland JM, Altman DG 1995 Multiple significance tests: the Bonferroni method BMJ 310:170

37. Franzese A, Vajro P, Argenziano A, Puzziello A, Iannucci MP, Saviano MC, Brunetti F, Rubino A 1997 Liver involvement in obese children. Ultrasonography and liver enzyme levels at diagnosis and during follow-up in an Italian population. Dig Dis Sci 42:1428-1432

38. Tominaga K, Kurata JH, Chen YK, Fujimoto E, Miyagawa S, Abe I, Kusano Y 1995 Prevalence of fatty liver in Japanese children and relationship to obesity. An epidemiological ultrasonographic survey. Dig Dis Sci 40:2002-2009

39. Wieckowska A, Feldstein AE 2008 Diagnosis of nonalcoholic fatty liver disease: invasive versus noninvasive. Semin Liver Dis 28:386-395

40. Targher G 2007 Non-alcoholic fatty liver disease, the metabolic syndrome and the risk of cardiovascular disease: the plot thickens. Diabet Med 24:1-6

41. Ioannou GN, Weiss NS, Boyko EJ, Mozaffarian D, Lee SP 2006 Elevated serum alanine aminotransferase activity and calculated risk of coronary heart disease in the United States. Hepatology 43:1145-1151

42. Adams LA, Lymp JF, St Sauver J, Sanderson SO, Lindor KD, Feldstein A, Angulo P 2005 The natural history of nonalcoholic fatty liver disease: a population-based cohort study. Gastroenterology 129:113-121

43. Ekstedt M, Franzen LE, Mathiesen UL, Thorelius L, Holmqvist M, Bodemar G, Kechagias S 2006 Long-term follow-up of patients with NAFLD and elevated liver enzymes. Hepatology 44:865-873

44. Matteoni CA, Younossi ZM, Gramlich T, Boparai N, Liu YC, McCullough AJ 1999 Nonalcoholic fatty liver disease: a spectrum of clinical and pathological severity. Gastroenterology 116:1413-1419

45. Brea A, Mosquera D, Martin E, Arizti A, Cordero JL, Ros E 2005 Nonalcoholic fatty liver disease is associated with carotid atherosclerosis: a case-control study. Arterioscler Thromb Vasc Biol 25:1045-1050

46. Targher G, Bertolini L, Rodella S, Tessari R, Zenari L, Lippi G, Arcaro G 2007 Nonalcoholic fatty liver disease is independently associated with an increased incidence of cardiovascular events in type 2 diabetic patients. Diabetes Care 30:2119-2121

47. Kim HC, Kim DJ, Huh KB 2009 Association between nonalcoholic fatty liver disease and carotid intima-media thickness according to the presence of metabolic syndrome. Atherosclerosis 204:521-525

48. Villanova N, Moscatiello S, Ramilli S, Bugianesi E, Magalotti D, Vanni E, Zoli M, Marchesini G 2005 Endothelial dysfunction and cardiovascular risk profile in nonalcoholic fatty liver disease. Hepatology 42:473-480

49. Pacifico L, Cantisani V, Ricci P, Osborn JF, Schiavo E, Anania C, Ferrara E, Dvisic G, Chiesa C 2008 Nonalcoholic fatty liver disease and carotid atherosclerosis in children. Pediatr Res 63:423-427

50. Demircioglu F, Kocyigit A, Arslan N, Cakmakçi H, Hizli S, Sedat AT 2008 Intima-media thickness of carotid artery and susceptibility to atherosclerosis in obese children with nonalcoholic fatty liver disease. J Pediatr Gastroenterol Nutr 47:68-75

51. Fabbrini E, Mohammed BS, Magkos F, Korenblat KM, Patterson BW, Klein S 2008 Alterations in adipose tissue and hepatic lipid kinetics in obese men and women with nonalcoholic fatty liver disease. Gastroenterology 134:424-431 